\title{
Wertheim's association theory applied to one site patchy colloids: Beyond the single bonding condition
}

\author{
Bennett D. Marshall, Deepti Ballal, and Walter G. Chapman ${ }^{\mathrm{a})}$ \\ Department of Chemical and Biomolecular Engineering, Rice University, 6100 S. Main Houston, \\ Texas 77005, USA
}

(Received 21 July 2012; accepted 23 August 2012; published online 14 September 2012; corrected 18 September 2012)

\begin{abstract}
We apply Wertheim's theory to develop an equation of state for one site patchy colloids where the patch can bond multiple times. We allow for the possibility of ring formation without the introduction of empirical parameters and show that for moderate patch coverage the infinite series of chain graphs is well represented by the first two terms. The theory is found to be in excellent agreement with new NVT and NPT Monte Carlo simulations. The approach described here can easily be converted to the form of a density functional theory to describe inhomogeneous patchy colloid systems. (C) 2012 American Institute of Physics. [http://dx.doi.org/10.1063/1.4751480]
\end{abstract}

\section{INTRODUCTION}

Primitive models for association have provided a route to model hydrogen bonding fluids for many decades. One such potential first introduced by Bohl ${ }^{1}$ and later used by Chapman et al. ${ }^{2,3}$ considers association as a square well interaction which depends on the position and orientation of each molecule. Kern and Frenkel ${ }^{4}$ later realized that this potential could describe the interaction between "patchy" colloids in a very precise way. Patchy colloids contain some number of localized attractive patches which results in orientation dependant interactions between spherical colloids; this is the physical realization of the primitive model for association introduced by Bohl. ${ }^{1}$ Patchy colloids have potential application for network forming fluids, ${ }^{5}$ empty liquids, ${ }^{6}$ and as a primitive model for proteins. ${ }^{7}$

A well suited statistical mechanical formalism to describe patchy systems is Wertheim's thermodynamic perturbation theory (TPT) ${ }^{8-12}$ In Wertheim's perturbation theory, orientation and steric effects are included at an early point in the theory allowing for accurate approximation schemes, such as TPT, where association is treated as a perturbation to a hard sphere reference fluid. In first order perturbation theory (TPT1) all contributions which account for a patch bonding more than once are neglected; this is Wertheim's single bonding condition. This is an exact graph cancellation for patches with small surface coverage, but as patch size increases the possibility of multiple bonds per patch must be accounted for. This is a problem recently addressed by Kalyuzhnyi et al. for the colloids with one ${ }^{13}$ or multiple ${ }^{14}$ attractive patches.

Kalyuzhnyi et $a l .{ }^{13}$ followed the methodology of Kalyuzhnyi and Stell ${ }^{15}$ and developed a multi-density theory for the one patch model where each bonding state of the patch was assigned a density. Within this formalism Kalyuzhnyi

\footnotetext{
a) Author to whom correspondence should be addressed. Electronic mail: wgchap@ rice.edu. Tel.: (1) 713.348.4900. Fax: (1) 713.348.5478.
}

et al. ${ }^{13}$ accounted for blocking effects (one colloid can block another colloid from approaching the patch) by enacting a resummed perturbation theory over an infinite series of chain graphs for colloids with a single patch where each patch can bond at most twice. A number of approximations were made in the resummation and the possibility of forming associated triatomic rings was neglected altogether. The resulting theory was an improvement over first order perturbation theory (TPT1) which does not allow for multiple patch association; however, to obtain good agreement with simulation data an empirical correction was added to the chain sum. All simulations were performed at low association energies (high temperatures) so the accuracy of this approach for strongly associating systems has yet to be verified.

In addition to bulk systems, Wertheim's theory has found wide application in density functional theories ${ }^{16-19}$ for inhomogeneous associating fluids. It would be highly desirable to develop a density functional theory for patchy colloids where multiple bonds per patch are allowed. The approach of Kalyuzhnyi et al. ${ }^{13}$ cannot be directly applied in inhomogeneous form; we must take another path.

In this work, we develop a new equation of state, in the form of a perturbation theory, for one patch colloids where multiple bonding per patch is allowed. We develop the theory in Wertheim's two density formalism for one site fluids. ${ }^{8}$ For simplicity, we will neglect all graphs where each patch is bonded more than twice. This is a rigorous cancellation for small to moderate patch coverage; for more extensive patch coverage the accuracy of this approximation will depend on maximum allowed bond angle, temperature, and density. We account for triatomic ring formation and develop an infinite sum of chain graphs to describe association into chains. We show that for moderate patch coverage, this infinite sum is well represented by the first two terms. To test the theory, we perform new NVT and NPT Monte Carlo simulations over a wide range of association energies. We show that the theory is in excellent agreement with simulation for moderate patch coverage even in strongly associating systems where 
TPT1 and the approach of Kalyuzhnyi et al. ${ }^{13}$ fail to give accurate predictions of bonding fractions and internal energy. For notational convenience, we make no attempt to keep density positional dependence in this work; however, a major advantage of the current approach is its applicability to inhomogeneous systems in the form of a density functional theory which will be the subject of a future publication.

\section{THEORY}

In this section, the equation of state will be developed for patchy colloids with a single patch. Here, we will work within Wertheim's two density formalism. ${ }^{8}$ The colloids consist of a hard core of diameter $\sigma$ with a single short range directional association patch. The potential of interaction between two colloids is given by

$$
\phi(12)=\phi_{H S}\left(r_{12}\right)+\phi_{A}(12) .
$$

The notation $(1) \equiv\left(\vec{r}_{1}, \Omega_{1}\right)$ represents the position $\vec{r}_{1}$ and orientation $\Omega_{1}$ of sphere $1, \phi_{R}\left(r_{12}\right)$ is the hard sphere potential

$$
\phi_{R}\left(r_{12}\right)=\left\{\begin{array}{cc}
\infty & r_{12}<\sigma \\
0 & \text { otherwise }
\end{array}\right.
$$

and $\phi_{A}(12)$ is the association potential of a conical site ${ }^{20}$

$$
\phi_{A}(12)=\left\{\begin{array}{cc}
-\varepsilon_{A}, & r_{12} \leq r_{c} ; \alpha_{1} \leq \alpha_{c} ; \alpha_{2} \leq \alpha_{c} \\
0 & \text { otherwise }
\end{array} .\right.
$$

Where $\vec{r}_{12}$ is the vector of magnitude $r_{12}$ connecting the centers of two colloids, $r_{c}$ is the maximum separation between two colloids for which association can occur, $\alpha_{1}$ is the angle between $\vec{r}_{12}$ and the orientation vector passing through the center of the patch on colloid 1 and $\alpha_{c}$ is the critical angle beyond which association cannot occur. Figure 1 gives a depiction of the association potential.

We will also use the Mayer functions $f_{A}(12)=\exp \left(-\phi_{A}(12)\right)-1$ and $f_{R}(12)=\exp$ $\left(-\phi_{R}(12)\right)-1$. The Helmholtz free energy is given as ${ }^{8}$

$$
\begin{aligned}
\frac{A-A_{R}}{k_{B} T}= & \int\left(\rho(1) \ln \frac{\rho_{o}(1)}{\rho(1)}+\rho(1)-\rho_{o}(1)\right) d(1) \\
& -c^{(o)}+c_{R}^{(o)} .
\end{aligned}
$$

Here $\rho(1)$ is the total sphere density at point (1) in the fluid, $\rho_{o}(1)$ is the monomer density, $A_{R}$ is the Helmholtz free energy of the hard sphere reference system, and $c^{(o)}$ is the fundamental graph sum

$$
\begin{aligned}
c^{(o)}= & \text { sum of all irreducible graphs consisting of monomer points } \\
& \text { carrying factors of } \rho, \text { s-mer subgraphs with } \mathrm{s} \geq 2 \text { and every } \\
& \text { point carrying a factor of } \rho_{\mathrm{o}}, \text { and } f_{R} \text {-bonds between some } \\
& \text { sets of points in distinct s-mers. }
\end{aligned}
$$

The term $c_{R}^{(o)}$ is the sum of all graphs which do not contain attraction bonds, then $\Delta c^{(o)}=c^{(o)}-c_{R}^{(o)}$ contains only graphs with attraction bonds. In the current work, we will employ Wertheim's single chain approximation and disregard all graphs with more than one path of attraction bonds. For $r_{c}=\sigma$, it is possible for a patch to associate at most once for $0 \leq \alpha_{c}<30^{\circ}$, twice for $30^{\circ} \leq \alpha_{c}<35.3^{\circ}, 3$ times for $35.3^{\circ} \leq \alpha_{c}<45^{\circ}$, and 4 times for $45^{\circ} \leq \alpha_{c}<58.3^{\circ} .{ }^{13}$ Here, we will only consider contributions to $\Delta c^{(o)}$ for patches bonded a maximum of twice. This is a rigorous graph cancellation for small $\alpha_{c}$. For larger $\alpha_{c}$, the accuracy of this approx-

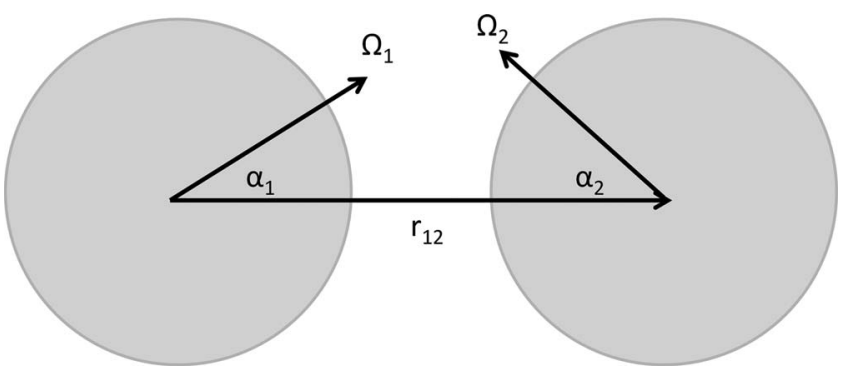

FIG. 1. Diagram of interacting colloids. imation will depend on $\alpha_{c}$, temperature (energy) and packing fraction $\eta$. Wertheim's theory can accommodate higher order interactions for patches bonded 3 and 4 times; one simply needs to include the contributions in $\Delta c^{(o)}$. With these approximations, we only to need consider 2 classes of graphs: the first are chain graphs which contain a single chain of attraction bonds for chains of length $2 \ldots \infty$, the second is the sum of all graphs with a single ring of attraction bonds between three spheres where each sphere is bonded twice

$$
\Delta c^{(o)}=\Delta c_{\text {ring }}^{(o)}+\sum_{n=2}^{\infty} \Delta c_{\mathrm{n}}^{(o)} .
$$

Where $\Delta c_{\mathrm{n}}^{(o)}$ is the sum of all chain graphs with a chain of $n-1$ attraction bonds. Employing the graphical definition of the $n$ body correlation function $g(1 \ldots n)$ the components to $\Delta c_{n}^{(o)}$ are approximated as

$$
\Delta c_{n}^{(o)} \approx \frac{1}{2} \int g_{R}(1 \ldots n) f_{A}(12) \ldots . . f_{A}(n-1, n) \prod_{\epsilon=1}^{n} \rho_{o}(\in) d(\in) .
$$

For the ring contribution, we follow an approach similar to Sear and Jackson who considered associated rings of spheres 
with 2 association sites. ${ }^{21}$ We consider all graphs with a single ring of three association bonds, with the graphical definition of $g(123)$ in mind we can approximate this infinite sum as

$$
\begin{aligned}
\Delta c_{\text {ring }}^{(o)} \approx & \frac{1}{6} \int \rho_{o}(1) f_{A}(12) \rho_{o}(2) f_{A}(23) \rho_{o}(3) f_{A}(13) \\
& \times g_{R}(123) d(1) d(2) d(3) .
\end{aligned}
$$

Minimizing the free energy with respect to monomer density, we obtain

$$
\rho(1)=\rho_{o}(1)+\rho_{1}(1)+\rho_{2 c}(1)+\rho_{\text {ring }}(1) .
$$

Where $\rho_{1}(1)$ is the density of colloids bonded once

$$
\begin{aligned}
\rho_{1}(1)= & \rho_{o}(1) \sum_{n=2}^{\infty} \int g_{R}(1 \ldots n) f_{A}(12) \ldots . . f_{A}(n-1, n) \\
& \times \prod_{\epsilon=2}^{n} \rho_{o}(\in) d(\epsilon),
\end{aligned}
$$

$\rho_{2 c}(1)$ is the density of colloids bonded twice in a chain

$$
\begin{aligned}
\rho_{2 c}(1)= & \rho_{o}(1) \sum_{n=2}^{\infty} \frac{n-2}{2} \int g_{R}(1 \ldots n) f_{A}(21) f_{A}(13) \ldots . f_{A} \\
& (n-1, n) \prod_{\epsilon=2}^{n} \rho_{o}(\in) d(\epsilon) .
\end{aligned}
$$

Finally, $\rho_{\text {ring }}(1)$ is the density of colloids which are in triatomic associated rings

$$
\begin{aligned}
\rho_{\text {ring }}(1)= & \frac{\rho_{o}(1)}{2} \int f_{A}(12) \rho_{o}(2) f_{A}(23) \rho_{o}(3) f_{A}(13) \\
& \times g_{R}(123) d(2) d(3) .
\end{aligned}
$$

Comparing (6), (8), (10), and (12),

$$
\begin{aligned}
\Delta c^{(o)} & =\int\left(\frac{\rho_{1}(1)}{2}+\frac{\rho_{\text {ring }}(1)}{3}\right) d(1) \\
& =\int \rho(1)\left(\frac{X_{1}(1)}{2}+\frac{X_{\text {ring }}(1)}{3}\right) d(1) .
\end{aligned}
$$

Where $X_{1}=\rho_{1} / \rho$ is the fraction of colloids bonded once, $X_{\text {ring }}$ $=\rho_{\text {ring }} / \rho$ is the fraction of colloids in triatomic rings, $X_{2 c}$ $=\rho_{2 c} / \rho$ is the fraction of colloids bonded twice in a chain, and $X_{o}=\rho_{o} / \rho$ is the fraction of monomers. Combining (4) and (13) and taking the homogeneous limit

$$
\frac{A-A_{R}}{N k_{B} T}=\ln X_{o}+1-X_{o}-\frac{X_{1}}{2}-\frac{X_{\text {ring }}}{3} .
$$

Define $X_{1}^{(n)}$ and $X_{2 c}^{(n)}$ as the fraction bonded once and twice in an $n$-mer chain. Clearly,

$$
\begin{aligned}
& X_{1}=\sum_{n=2}^{\infty} X_{1}^{(n)}, \\
& X_{2 c}=\sum_{n=3}^{\infty} X_{2 c}^{(n)} .
\end{aligned}
$$

From Eq. (10),

$$
\begin{aligned}
X_{1}^{(n)}= & \rho^{n-1}\left(X_{o}\right)^{n} \int g_{R}(1 \ldots n) \frac{f_{A}(12)}{\Omega} \ldots \\
& \times \frac{f_{A}(n-1, n)}{\Omega} d(2) \ldots d(n),
\end{aligned}
$$

where $\rho=\rho(1) \Omega=4 \pi \rho$ (1). From Eq. (11),

$$
X_{2 c}^{(n)}=\frac{(n-2)}{2} X_{1}^{(n)} .
$$

Combining (14) and (15), we obtain the final form for the free energy

$$
\frac{A-A_{R}}{N k_{B} T}=\ln X_{o}+1-X_{o}-\frac{X_{\text {ring }}}{3}-\sum_{n=2}^{\infty} \frac{X_{1}^{(n)}}{2} .
$$

Instead of attempting an approximate summation of all chain graphs given in Eq. (18), we will show that keeping the first 2 contributions to the sum yields a good description of the thermodynamic behavior of the system. We believe the neglect of longer chain graphs is reasonable for critical angles $\alpha_{c}$ which are not too large; for instance, it is much more difficult to position and orient 4 colloids to associate into a 4-mer chain than it is to position and orient 3 into a triatomic chain. As association energies increase the enthalpic benefit of association would overcome this entropic penalty; however, the presence of triatomic rings should temper this effect due to the fact that at high association energies the triatomic rings should dominate. For instance, in the limit of infinitely strong association $f_{A} \rightarrow \infty$ and $\rho_{o} \rightarrow 0$; then $\Delta c_{\text {ring }}^{(o)} \sim \rho_{o}^{3} f_{A}^{3}$ so $\rho_{o} \sim f_{A}^{-1}$ and $\Delta c_{n}^{(o)} \sim \rho_{o}^{n} f_{A}^{n-1} \sim f_{A}^{-1}=0$ which shows that for infinitely strong association only triatomic rings remain. Of course, for large $\alpha_{c}$ this is an artificial limit, at some point the neglected graphs accounting for colloids bonded more than twice would become significant and would have to be accounted for. With these arguments in mind, we truncate the sum in Eq. (15) at $n=3$,

$$
\begin{aligned}
& X_{1} \approx X_{1}^{(2)}+X_{1}^{(3)}, \\
& X_{2 c} \approx X_{2 c}^{(3)} .
\end{aligned}
$$

The free energy is then

$$
\frac{A-A_{R}}{N k_{B} T} \approx \ln X_{o}+1-X_{o}-\frac{X_{\text {ring }}}{3}-\frac{X_{1}^{(2)}}{2}-\frac{X_{1}^{(3)}}{2} .
$$

Now we eliminate the $g$ correlation functions in favor of cavity correlation functions $e_{R}(r) y_{R}(r)=g_{R}(r)$ and $e_{R}\left(r_{12}\right) e_{R}\left(r_{23}\right) e_{R}\left(r_{13}\right) y_{R}\left(r_{12}, r_{23}, r_{13}\right)=g_{R}\left(r_{12}, r_{23}, r_{13}\right)$ with $e_{R}(r)=\exp \left[-\phi_{R}(r) / k_{B} T\right]$. We approximate the triplet cavity correlation function in $X_{1}^{(3)}$ and $X_{\text {ring }}$ as

$$
y_{R}\left(r_{12}, r_{23}, r_{13}\right) \approx y_{R}\left(r_{12}\right) y_{R}\left(r_{23}\right) y_{o}\left(r_{12}, r_{23}, r_{13}\right) \text {. }
$$

The term $y_{o}\left(r_{12}, r_{23}, r_{13}\right)$ is a correction to the linear superposition. Since the colloids which are bonded with a $y_{R}(r)$ also share an association bond, they are near hard sphere contact and we can employ the approximation ${ }^{2,3}$

$$
r^{2} y_{R}(r) \approx \sigma^{2} y_{R} .
$$

Where $y_{R}$ is the cavity correlation function at hard sphere contact. For $y_{o}\left(r_{12}, r_{23}, r_{13}\right)$, we employ the fitting function 


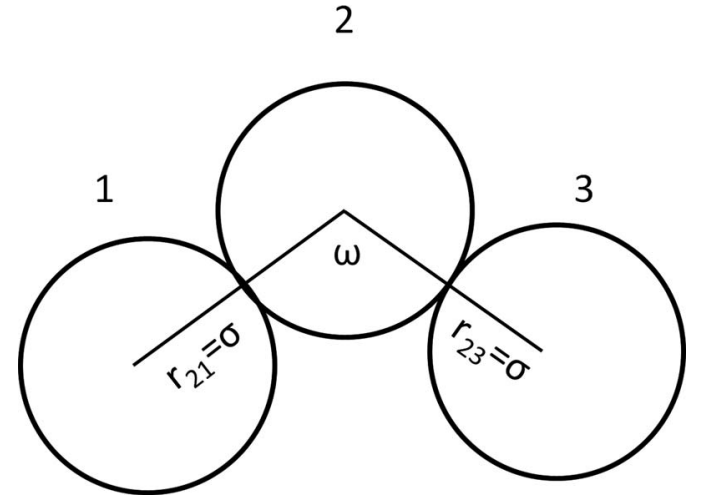

FIG. 2. Diagram of three interacting colloids where colloids 1 and 3 are both in contact with colloid 2.

of Müller and Gubbins ${ }^{22}$ for three hard spheres in rolling contact

$$
y_{o}\left(r_{12}, r_{23}, r_{13}\right) \approx y_{o}\left(\sigma, \sigma, 2 \sin \left(\bar{\omega}_{i} / 2\right)\right) \equiv y_{o}\left(\bar{\omega}_{i}\right) .
$$

Here, $\bar{\omega}_{i}=\bar{\omega}_{i}\left(\alpha_{c}\right)$ is the average angle (similar to bond angle) between the first and third colloids in the chain or ring ( $i=$ chain, ring) when colloids 1 and 3 are both in contact with colloid 2, see Fig. 2 for diagram. The function $y_{o}\left(\bar{\omega}_{i}\right)$ is given as

$$
y_{o}\left(\bar{\omega}_{i}\right)=\frac{1+a \eta+b \eta^{2}}{(1-\eta)^{3}} .
$$

Where $\eta=\frac{\pi}{6} \rho \sigma^{3}$ is the packing fraction, the constants $a$ and $b$ depend on bond angle (average angle $\bar{\omega}$ here) and are determined by fitting Eq. (24) to the tabulated results ${ }^{23}$ of
Attard's PY3 theory. ${ }^{24,25}$ The constants $a$ and $b$ are tabulated in Ref. 22.

as

Using Eqs. (21) and (24), we can simplify $X_{1}^{(2)}$ and $X_{1}^{(3)}$

$$
\begin{gathered}
X_{1}^{(2)}=\pi \sigma^{2}\left(r_{c}-\sigma\right)\left(1-\cos \alpha_{c}\right)^{2} \rho y_{R} f_{A} X_{o}^{2}, \\
X_{1}^{(3)}=\rho^{2} f_{A}^{2} \sigma^{6} y_{R}^{2} y_{o}\left(\bar{\omega}_{\text {chain }}\right) \Gamma X_{o}^{3} .
\end{gathered}
$$

Where the integral $\Gamma$ is a geometric quantity given by

$$
\begin{aligned}
\Gamma= & \frac{1}{\Omega^{3} V \sigma^{2}} \int \frac{\lambda\left(r_{12}\right)}{r_{12}^{2}} \frac{\lambda\left(r_{23}\right)}{r_{23}^{2}} e_{R}\left(r_{13}\right) U\left(\Omega_{1}, \Omega_{2}\right) \\
& \times U\left(\Omega_{2}, \Omega_{3}\right) d(1) d(2) d(3) .
\end{aligned}
$$

Where

$$
\lambda\left(r_{12}\right)=\left\{\begin{array}{cc}
1 & \sigma \leq r_{12} \leq r_{c} \\
0 & \text { otherwise }
\end{array}\right.
$$

and

$$
U\left(\Omega_{1}, \Omega_{2}\right)=\left\{\begin{array}{cc}
1 & \alpha_{1} \leq \alpha_{c} \text { and } \alpha_{2} \leq \alpha_{c} \\
0 & \text { otherwise }
\end{array} .\right.
$$

The fraction of segments in triatomic rings is

$$
X_{\text {ring }}=\frac{1}{2} f_{A}^{3} \Psi \sigma^{6} y_{R}^{2} y_{o}\left(\bar{\omega}_{\text {ring }}\right) X_{o}^{3} \rho^{2} .
$$

Where the geometric integral $\Psi$ is given by

$$
\begin{aligned}
\Psi= & \frac{1}{\Omega^{3} V \sigma^{2}} \int \frac{\lambda\left(r_{12}\right)}{r_{12}^{2}} \frac{\lambda\left(r_{23}\right)}{r_{23}^{2}} \lambda\left(r_{13}\right) U\left(\Omega_{1}, \Omega_{2}\right) \\
& \times U\left(\Omega_{2}, \Omega_{3}\right) U\left(\Omega_{1}, \Omega_{3}\right) d(1) d(2) d(3) .
\end{aligned}
$$

The average angle $\bar{\omega}_{\text {chain }}$ is obtained from the inverse cosine of the average

$$
\overline{\cos \omega_{\text {chain }}}=\frac{\int \cos \omega_{\text {chain }} \delta\left(r_{12}-\sigma\right) \delta\left(r_{23}-\sigma\right) e_{R}\left(r_{13}\right) U\left(\Omega_{1}, \Omega_{2}\right) U\left(\Omega_{2}, \Omega_{3}\right) d(1) d(2) d(3)}{\int \delta\left(r_{12}-\sigma\right) \delta\left(r_{23}-\sigma\right) e_{R}\left(r_{13}\right) U\left(\Omega_{1}, \Omega_{2}\right) U\left(\Omega_{2}, \Omega_{3}\right) d(1) d(2) d(3)} .
$$

Where $\delta(x)$ is the Dirac delta function. Similarly for $\bar{\omega}_{\text {ring }}$, we have

$$
\overline{\cos \omega_{\text {ring }}}=\frac{\int \cos \omega_{\text {ring }} \delta\left(r_{12}-\sigma\right) \delta\left(r_{23}-\sigma\right) \lambda\left(r_{13}\right) U\left(\Omega_{1}, \Omega_{2}\right) U\left(\Omega_{2}, \Omega_{3}\right) U\left(\Omega_{1}, \Omega_{3}\right) d(1) d(2) d(3)}{\int \delta\left(r_{12}-\sigma\right) \delta\left(r_{23}-\sigma\right) \lambda\left(r_{13}\right) U\left(\Omega_{1}, \Omega_{2}\right) U\left(\Omega_{2}, \Omega_{3}\right) U\left(\Omega_{1}, \Omega_{3}\right) d(1) d(2) d(3)} .
$$

Table I list numerical Monte Carlo calculations for $\Gamma, \Psi$, $\bar{\omega}_{\text {ring }}$, and $\bar{\omega}_{\text {chain. }}$. Clearly from Eqs. (9) and (17), we have the condition

$$
X_{o}+X_{1}^{(2)}+\frac{3}{2} X_{1}^{(3)}+X_{\text {ring }}=1
$$

Wertheim provides the Euler-Lagrange equation ${ }^{8}$

$$
\begin{aligned}
\rho_{o}(1) & =z \exp \left(c_{o}(1)\right) \\
& =\exp \left(\frac{\mu}{k_{B} T}-\frac{\delta A_{R} / k_{B} T}{\delta \rho(1)}+\frac{\delta \Delta c^{(o)}}{\delta \rho(1)}\right) .
\end{aligned}
$$

From which we obtain the chemical potential $\mu$,

$$
\begin{aligned}
\frac{\mu}{k_{B} T}= & \frac{\mu_{R}}{k_{B} T}+\ln X_{o}-\left(\frac{X_{1}}{2}+\frac{X_{\text {ring }}}{3}\right) \eta \frac{\partial \ln y_{R}}{\partial \eta} \\
& -\left(X_{2}-X_{\text {ring }}\right) \eta \frac{\partial \ln \left(y_{R} \times y_{o}\left(\bar{\omega}_{\text {chain }}\right)\right)}{\partial \eta} \\
& -\frac{X_{\text {ring }}}{3} \eta \frac{\partial \ln \left(y_{R} \times y_{o}\left(\bar{\omega}_{\text {ring }}\right)\right)}{\partial \eta} .
\end{aligned}
$$

The fraction $X_{2}=X_{2 c}+X_{\text {ring }}$ is the fraction of colloids bonded twice. The pressure and internal energy can then be 
TABLE I. Numerical results for geometric integrals $\Gamma$ and $\Psi$ and angles $\bar{\omega}_{\text {chain }}$ and $\bar{\omega}_{\text {ring }}$.

\begin{tabular}{ccccc}
\hline \hline$\alpha_{\mathrm{c}}$ & $\Gamma$ & $\Psi$ & $\bar{\omega}_{\text {chain }}$ & $\bar{\omega}_{\text {ring }}$ \\
\hline 25 & 0 & 0 & & \\
30 & $8.47 \times 10^{-8}$ & 0 & $60^{\circ}$ & \\
35 & $4.88 \times 10^{-6}$ & $1.52 \times 10^{-8}$ & $65.1^{\circ}$ & $65.5^{\circ}$ \\
40 & $3.62 \times 10^{-5}$ & $4.46 \times 10^{-7}$ & $69.3^{\circ}$ & $65.8^{\circ}$ \\
45 & $1.49 \times 10^{-4}$ & $3.19 \times 10^{-6}$ & $72.7^{\circ}$ & $65.9^{\circ}$ \\
50 & $4.56 \times 10^{-4}$ & $1.29 \times 10^{-5}$ & $75.7^{\circ}$ & $65.9^{\circ}$ \\
55 & $1.16 \times 10^{-3}$ & $3.88 \times 10^{-5}$ & $78.1^{\circ}$ & $65.9^{\circ}$ \\
\hline \hline
\end{tabular}

obtained through the relations

$$
\begin{gathered}
\frac{P}{\rho k_{B} T}=\frac{\mu}{k_{B} T}-\frac{A}{N k_{B} T}, \\
E=\frac{\partial(\beta A / N)}{\partial \beta}=\left(\frac{1}{X_{o}}-1\right) \frac{\partial X_{o}}{\partial \beta}-\frac{1}{2} \frac{\partial X_{1}}{\partial \beta}-\frac{1}{3} \frac{\partial X_{\text {ring }}}{\partial \beta} .
\end{gathered}
$$

Equation (38) completes the homogeneous theory for one patch colloids where each patch can bond a maximum of two times. We have allowed for the colloids to associate into diatomic and triatomic chains as well as triatomic rings and neglected the association into longer $n$-mers. We will validate the assumptions made in the model by comparison to simulation data in Sec. IV.

\section{SIMULATIONS}

To validate the new theory, Monte Carlo simulations were performed in the $N V T$ ensemble (constant number of colloids $N$, volume $V$, and temperature $T$ ) and $N P T$ ensemble (constant number of colloids $N$, pressure $P$, and temperature $T$ ) for colloids which interact through the potential given in Eq. (1) with the cutoff radius chosen as $r_{c}=1.1 \sigma$. In this work, we use $N=256$ colloids.

$N V T$ simulations were carried out using the standard Metropolis algorithm ${ }^{26}$ where each new configuration is generated by an attempted displacement and reorientation (trial move) of a single colloid. Each simulation begins with all 256 colloids in a face centered cubic lattice; the simulation is then allowed to equilibrate for $10^{6}$ cycles at which point averages are taken over an additional $10^{6}$ production cycles. Here, a cycle is defined as $N$ trial moves. The angle change and displacement parameters were modified to obtain acceptance ratios of $30 \%-40 \%$.

$N P T$ simulations were performed in the same manner as the NVT simulations with the addition of an attempted volume change for each $N$ attempted trial moves. The volume displacement parameter was chosen to yield volume change acceptance ratios of approximately $10 \%$.

\section{RESULTS}

In this section, we validate the theory derived in Sec. II by comparison to new Monte Carlo simulations. Our goal is to test the assumptions used in the development of the theory and determine the range of validity. We begin (Figure 3)

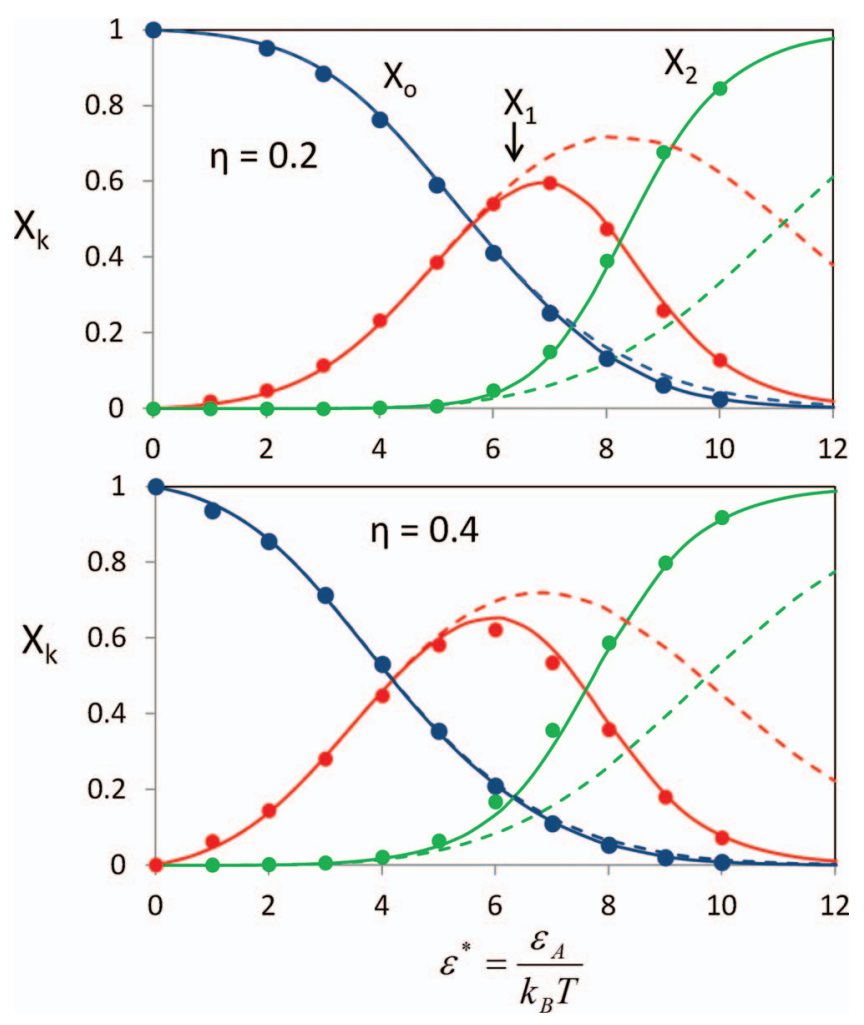

FIG. 3. Comparison of theoretical (current theory - solid lines, theory of Kalyuzhnyi et al. ${ }^{13}$ - dashed lines), and simulation (symbols) predictions for the monomer fractions $X_{o}$ (blue), fraction of colloids bonded once $X_{1}=$ $X_{1}^{(2)}+X_{1}^{(3)}$ (red) and fraction bonded twice $X_{2}=X_{2 c}^{(3)}+X_{\text {ring }}$ (green) at packing fractions of $\eta=0.2$ top and $\eta=0.4$ bottom; each with $\alpha_{c}=35^{\circ}$.

by considering the various bonding fractions (monomer $X_{o}$, bonded once $-X_{1}$, and bonded twice $-X_{2}=X_{\text {ring }}$ $\left.+X_{2 c}\right)$ as function of reduced association energy $\varepsilon^{*}=\varepsilon_{A} / k_{B} T$ at packing fractions $\eta=0.2$ and 0.4 . The critical angle is set to $\alpha_{c}=35^{\circ}$ so the probability of a patch bonding more than twice is not significant. The symbols are simulation data and solid curves give the predictions of the current theory. For comparison, the dashed curves give theoretical predictions using the resummed perturbation theory of Kalyuzhnyi et al. ${ }^{13}$ At each $\eta$ increasing $\varepsilon^{*}$ increases the degree of association. At low $\varepsilon^{*}, X_{1}$ is larger than $X_{2}$ due to the fact that the entropic penalty of positioning and orienting two colloids for association is small compared to the penalty paid to position and orient multiple colloids into larger clusters. As $\varepsilon^{*}$ increases the energetic benefit of forming multiple bonds overcomes this entropic penalty until $X_{2}$ dominates at large $\varepsilon^{*}$. This energetic - entropic tug of war results in a maximum in $X_{1}$ whose location increases with decreasing packing fraction. In each case, the current theory and simulation are in excellent agreement; the resumed perturbation theory of Kalyuzhnyi et al. ${ }^{13}$ fails to accurately predict $X_{1}$ and $X_{2}$ for $\varepsilon^{*}>5$.

A major assumption in the development of the theory was that for moderate $\alpha_{c}$ the infinite sum of chain diagrams Eq. (15) could be truncated for $n>3$. To test this assumption, in Fig. 4, we compare theoretical and simulation predictions of the fraction of colloids bonded twice in triatomic rings $X_{\text {ring }}$ and the fraction bonded twice in chains $X_{2 c}$ with $\alpha_{c}=35^{\circ}$ at 


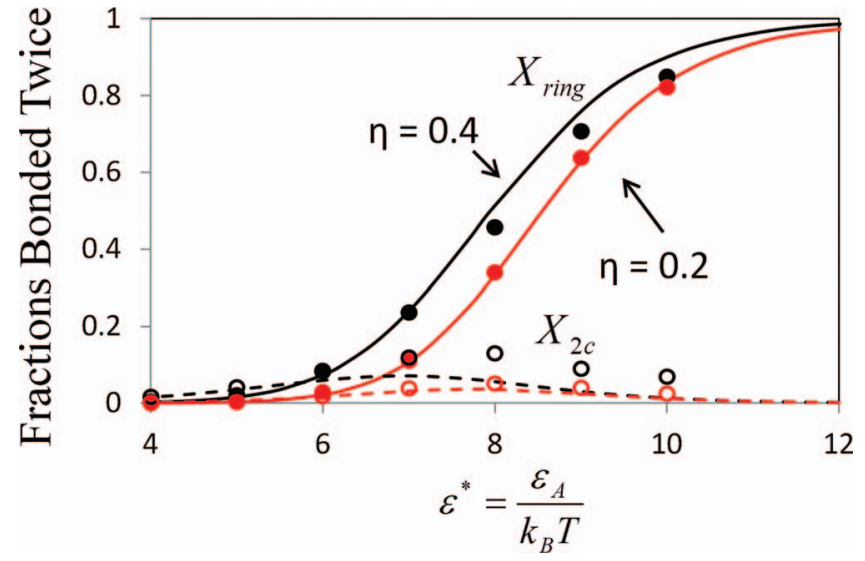

FIG. 4. Fractions bonded twice in a ring $X_{\text {ring }}$ (solid lines - theory, filled symbols - simulation) and twice in a chain $X_{2 c}$ (dashed line - theory, open symbols - simulation) at packing fractions of $\eta=0.2$ (red) and $\eta=0.4$ (black); for each case $\alpha_{c}=35^{\circ}$.

packing fractions $\eta=0.2$ and 0.4. At low $\varepsilon^{*}, X_{2 c}$ dominates due to the fact that the entropic penalty of positioning and orienting 3 colloids to bond in a chain is less than that paid to form a triatomic ring; however, upon increasing $\varepsilon^{*}$ this entropic penalty is rapidly overtaken by the energetic benefit of forming the triatomic rings with higher density favoring ring formation. Overall the theory and simulation are in fair agreement. The theory is accurate for $\eta=0.2$, but at $\eta=0.4$ predicts values of $X_{\text {ring }}$ which are a little too large and predicts values of $X_{2 c}$ which are a little too low; this is a result of the neglect of all chain graphs for $n>3$. That said, the truncation of the infinite sum for $n>3$, Eq. (19), is a good approximation for $\alpha_{c}=35^{\circ}$ over the full range of $\varepsilon^{*}$. For large $\alpha_{c}$ at high $\varepsilon^{*}$ this truncation will fail.

Now in Fig. 5, we compare simulation and theoretical predictions for the reduced energy per molecule $E^{*}=E / N k_{B} T$ as a function of $\varepsilon^{*}$ for $\alpha_{c}=35^{\circ}$ and packing fractions $\eta=0.2$ and 0.4. For comparison, we have included TPT1 predictions for $E^{*}$ where only 1 bond per patch is allowed; we have also included theoretical predictions from the resummed perturbation theory of Kalyuzhnyi et al. ${ }^{13}$ TPT1 (short dashed curve) significantly under predicts the magnitude of $E^{*}$ due to the fact that the possibility of 2 bonds per patch is not accounted for. The equation of state of Kalyuzhnyi et al. ${ }^{13}$ (long dashed curve) is accurate for $\varepsilon^{*}<5$, but underpredicts the magnitude of the energy at higher $\varepsilon^{*}$. Our theory Eq. (38) (solid curve) is in excellent agreement with the simulation data over the full range of $\varepsilon^{*}$. Increasing density increases the magnitude $E^{*}$ for moderate $\varepsilon^{*}$; however, for very strong association $E^{*}$ becomes independent of density.

A key approximation made here and in the work of Kalyuzhnyi et al. ${ }^{13}$ is the neglect of all graphs which account for patches bonded more than twice. This is a rigorous graph cancellation for small $\alpha_{c}$; however, for larger $\alpha_{c}$ the neglect of these higher order graphs is an approximation whose accuracy will depend on $\varepsilon^{*}, \alpha_{c}$, and $\eta$. In Fig. 6, we compare theoretical and simulation predictions of $E^{*}$ as a function of $\alpha_{c}$ at a packing fraction of $\eta=0.3$ for a low association energy of $\varepsilon^{*}=3$ and a moderate $\varepsilon^{*}=5$; for comparison, we have included predictions from TPT1. For the weakly associating system at $\varepsilon^{*}$
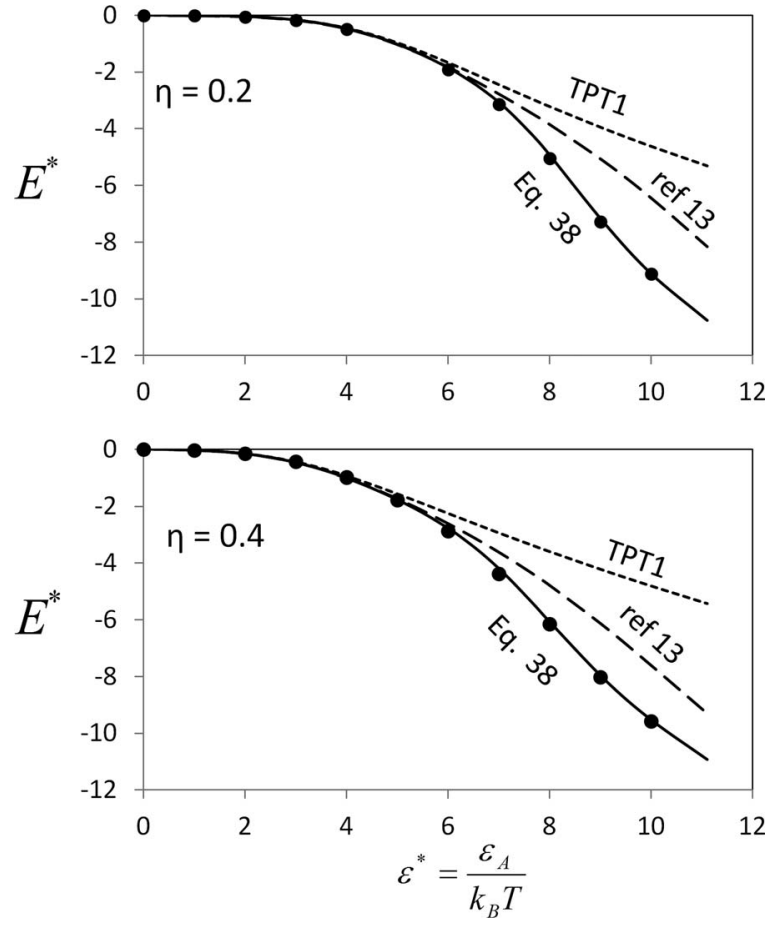

FIG. 5. Energy per colloid $E^{*}=E / N k_{B} T$ at a critical angle $\alpha_{c}=35^{\circ}$ and packing fractions $\eta=0.2$ (top) and $\eta=0.4$ (bottom). Symbols are simulation results, solid line is from the current theory Eq. (38), long dashed line is predictions from the theory of Kalyuzhnyi et al., ${ }^{13}$ and short dashed line is TPT1 predictions.

$=3$, the current theory is in good agreement with simulation data over the full range of $\alpha_{c}$, while TPT1 under predicts the magnitude of $E^{*}$. When $\varepsilon^{*}$ is increased to $\varepsilon^{*}=5$, the current theory is in excellent agreement with simulation for $\alpha_{c} \leq 40^{\circ}$; for larger association energies the theory fails. The genesis of this failure is illustrated by the inset figure which shows the rapid increase in the fraction of colloids bonded 3 times $X_{3}$ for $\alpha_{c}>40^{\circ}$. If the theory is to be extended to account for these higher order interactions additional graphs must be added to the graph sum $\Delta c^{(o)}$.

Lastly, in Fig. 7, we compare predictions of the change in pressure due to association $\Delta P^{*}=\left(P-P_{H S}\right) \sigma^{3} / k_{B} T$ using the current theory (solid curves) and the theory of Kalyuzhnyi

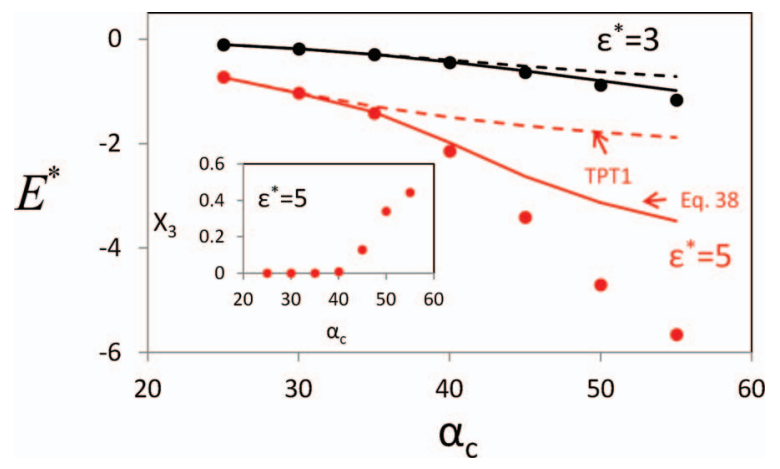

FIG. 6. Energy per colloid $E^{*}=E / N k_{B} T$ as a function of critical angle $\alpha_{c}$. The packing fraction is fixed at $\eta=0.3$, symbols give simulation results, solid curves give current theory predictions Eq. (38), and dashed curves are TPT1 predictions. Color code: red represents $\varepsilon^{*}=5$ and black represents $\varepsilon^{*}$ $=3$. Inset gives the simulated fraction of colloids bonded 3 times $X_{3}$. 

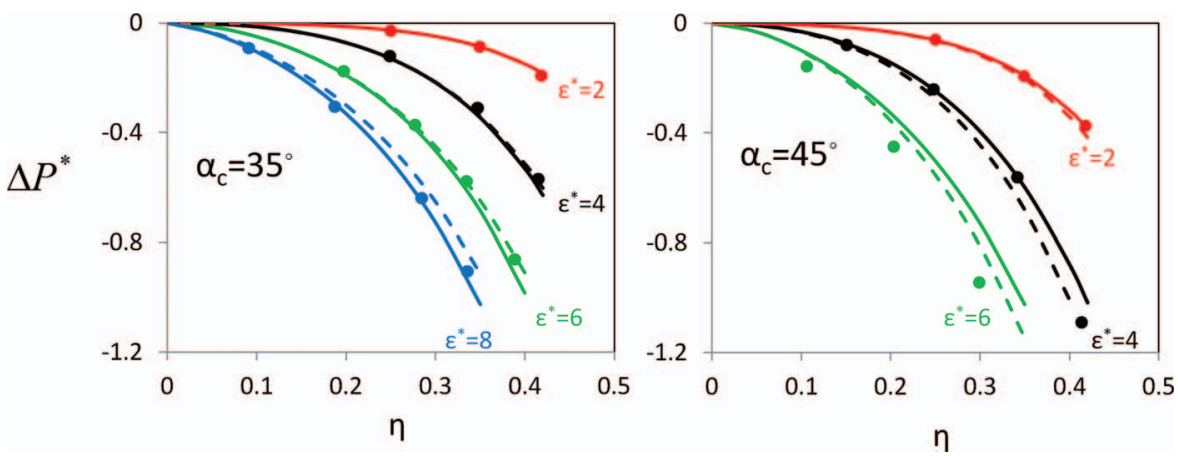

FIG. 7. Change in pressure due to association $\Delta P^{*}$ for $\alpha_{c}=35^{\circ}$ (left) and $\alpha_{c}=45^{\circ}$ (right). Symbols give $N P T$ simulation results, solid curves give theoretical predictions from the current work and dashed curves give theoretical predictions using the theory of Ref. 13 .

et al. ${ }^{13}$ (dashed curves) to new NPT simulation results for critical angles $\alpha_{c}=35^{\circ}$ and $45^{\circ}$. Both theories are in good agreement with simulation data for $\alpha_{c}=35^{\circ}$ with the current theory being most accurate at high energy. At this critical angle, the current theory predicts a slightly stronger response to association than the theory of Kalyuzhnyi et al. ${ }^{13}$ At $\alpha_{c}=45^{\circ}$, each theory is in good agreement with simulation data for $\varepsilon^{*}=2$ and the current theory is accurate for $\varepsilon^{*}=4$ (except at the highest density). For $\varepsilon^{*}=6$, each theory predicts too weak of a pressure response to association; at this $\varepsilon^{*}$ the fraction of colloids bonded three times $X_{3}$ becomes significant (see Fig. 6), since neither theory accounts for colloids bonded more than twice they underpredict the pressure response to association. The current theory predicts a weaker pressure response to association than the theory of Kalyuzhnyi et al. ${ }^{13}$ at this $\alpha_{c}$.

\section{CONCLUSIONS}

In Wertheim's two density formalism, we have developed a simple equation of state for colloids with a single association patch. We included the possibility of triatomic ring formation and truncated the contributions of associated chains at $n=3$. By comparison to new NVT and NPT Monte Carlo simulations, it was shown that this truncation is a good approximation. At $\alpha_{c}=35^{\circ}$, the theory performed very well in comparison to simulation over the full range of $\varepsilon^{*}$, while TPT1 and the approach of Kalyuzhnyi et al. ${ }^{13}$ fail to give accurate predictions of bonding fractions and internal energy in strongly associating systems. Also, we neglected all graphs involving patches bonded more than twice; these graphs are negligible for moderate $\alpha_{c}$. For larger $\alpha_{c}$, the error incurred by neglect of these graphs will increase with increasing $\varepsilon^{*}$ and $\eta$. Wertheim's two density formalism can account for higher order interactions (bonded more than twice), we simply need to include the relevant graphs. That said, for very large $\alpha_{c}$ a perturbation theory will likely be insufficient and integral equation theory ${ }^{27}$ may be required. In its range of validity, the current theory has the distinct advantage of simplicity over integral equation theory. Another advantage of our approach is that the extension of the current methodology to inhomogeneous systems in the form of a density functional theory is straightforward. This will be the subject of our next publication.
Finally, in this work our focus was on one patch colloids; however, the general approach taken here should be easy to extend to the multi-patch case using Wertheim's multi-density formalism. ${ }^{10}$ This is a problem we shall address in a future publication.

\section{ACKNOWLEDGMENTS}

The financial support for this work was provided by the Robert A. Welch Foundation (Grant No. C-1241) and by the National Science Foundation (CBET - 0756166).

${ }^{1}$ W. Bohl, Mol. Phys. 45, 605 (1982).

${ }^{2}$ W. G. Chapman, Ph.D. dissertation, Coronell University, Ithaca, NY, 1988.

${ }^{3}$ G. Jackson, W. G. Chapman, and K. E. Gubbins, Mol. Phys. 65(1), 1-31 (1988).

${ }^{4}$ N. Kern and D. Frenkel, J. Chem. Phys. 118, 9882 (2003).

${ }^{5}$ D. de las Heras, J. M. Tavares, and M. M. T. da Gama, Soft Matter 7(12), 5615-5626 (2011)

${ }^{6}$ E. Bianchi, J. Largo, P. Tartaglia, E. Zaccarelli, and F. Sciortino, Phys. Rev. Lett. 97(16), 168301 (2006).

${ }^{7}$ E. Bianchi, R. Blaak, and C. N. Likos, Phys. Chem. Chem. Phys. 13(14), 6397-6410 (2011)

${ }^{8}$ M. Wertheim, J. Stat. Phys. 35(1), 19-34 (1984).

${ }^{9}$ M. Wertheim, J. Stat. Phys. 35(1), 35-47 (1984).

${ }^{10}$ M. Wertheim, J. Stat. Phys. 42(3), 459-476 (1986).

${ }^{11}$ M. Wertheim, J. Stat. Phys. 42(3), 477-492 (1986).

${ }^{12}$ M. Wertheim, J. Chem. Phys. 87, 7323 (1987).

${ }^{13}$ Y. Kalyuzhnyi, H. Docherty, and P. Cummings, J. Chem. Phys. 133, 044502 (2010).

${ }^{14}$ Y. Kalyuzhnyi, H. Docherty, and P. Cummings, J. Chem. Phys. 135, 014501 (2011).

${ }^{15}$ Y. V. Kalyuzhnyi and G. Stell, Mol. Phys. 78(5), 1247-1258 (1993).

${ }^{16}$ A. Bymaster and W. Chapman, J. Phys. Chem. B 114(38), 12298-12307 (2010).

${ }^{17}$ B. D. Marshall, A. J. García-Cuéllar, and W. G. Chapman, J. Chem. Phys. 136, 154103 (2012)

${ }^{18}$ B. D. Marshall, A. J. García-Cuéllar, and W. G. Chapman, "A perturbation density functional theory for hydrogen bonding cyclic molecules," Mol. Phys. (in press).

${ }^{19}$ C. J. Segura, W. G. Chapman, and K. P. Shukla, Mol. Phys. 90(5), 759-771 (1997).

${ }^{20}$ W. G. Chapman, G. Jackson, and K. E. Gubbins, Mol. Phys. 65(5), 10571079 (1988).

${ }^{21}$ R. Sear and G. Jackson, Phys. Rev. E 50(1), 386-394 (1994).

${ }^{22}$ E. Müller and K. Gubbins, Mol. Phys. 80(4), 957-976 (1993).

${ }^{23}$ P. Attard and G. Stell, Chem. Phys. Lett. 189(2), 128-132 (1992).

${ }^{24}$ P. Attard, J. Chem. Phys. 91, 3072 (1989).

${ }^{25}$ P. Attard, J. Chem. Phys. 91, 3083 (1989).

${ }^{26}$ N. Metropolis, A. W. Rosenbluth, M. N. Rosenbluth, A. H. Teller, and E. Teller, J. Chem. Phys. 21(6), 1087 (1953).

${ }^{27}$ A. Giacometti, F. Lado, J. Largo, G. Pastore, and F. Sciortino, J. Chem. Phys. 131(17), 174114 (2009). 